\title{
Crystal structure of defect pentavanadium tetratelluride, $\mathbf{V}_{\mathbf{4 . 6 4}} \mathbf{T e}_{4}$
}

\author{
Th. E. Weirich, R. Pöttgen and A. Simon
}

Max-Planck-Institut für Festkörperforschung, Heisenbergstr. I. D-70569 Stuttgart, Germany

Received November 13, 1996, transferred to Ist update of database ICSD in 1998, CSD-No. 402709

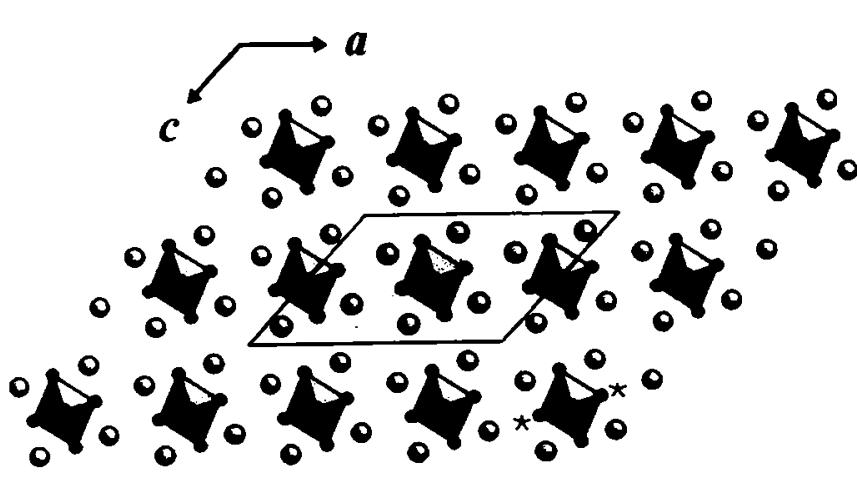

Fig. 1. Projection along the short $b$ axis with emphasis on the condensed $V_{6}$ octahedra $(V(2)=*)$.

Source of material: Powders of vanadium and tellurium $(5: 4$ atomic ratio) were pre-reacted in an alumina crucible at $1370 \mathrm{~K}$ under vacuum, subsequently pressed to a pellet in a dry-box and inductively heated close to melting under purified argon for two hours. The pellet was cooled by radiative heat loss and single crystals were selected from the sample by mechanical fragmentation.

$\mathrm{V}_{4.64} \mathrm{Te}_{4}$ is isotypic with monoclinic $\mathrm{Nb}_{4.7} \mathrm{Te}_{4}$ (see ref. 1). The title compound was first reported by Grönvold, Hagberg and Haraldsen (see ref. 2) with the tentative composition $V_{5} T_{4} ; a=1348 \mathrm{pm}$, $b=391 \mathrm{pm}, c=1382 \mathrm{pm}$ and $\beta=93.5^{\circ}$ from $X$-ray film data, possible space groups $\mathrm{Cc}$ or $\mathrm{C} 2 / \mathrm{c}$. The present structure refinement from single-crystal diffractometer data showed that the correct composition is $\mathrm{V}_{4.64} \mathrm{Te}_{4}$ in agreement with our previous study on the niobium compound. The structure of $\mathrm{V}_{4.64 \mathrm{Te}_{4}}$ is closely related to that of $\mathrm{TisTe}_{4}$ (see ref. 3). It is built from strings of compressed $\mathrm{V}_{6}$-octahedra along [010] which are surrounded by the tellurium atoms like in the $\mathrm{M}_{6} \mathrm{X}_{8}$ type cluster. The $\mathrm{V}(2)$ position within the octahedra is occupied by $82(1) \%$ only.

Table 2. Final atomic coordinates and displacement parameters (in $\AA^{2}$ )

\begin{tabular}{lllllllllll}
\hline Aiom & Site & $x$ & $y$ & $z$ & $U_{11}$ & $U_{22}$ & $U_{33}$ & $U_{12}$ & $U_{13}$ & $U_{23}$ \\
\hline $\mathrm{V}(1)$ & $2 c$ & 0 & 0 & $1 / 2$ & $0.004(1)$ & $0.015(2)$ & $0.006(1)$ & 0 & $0.002(1)$ & 0 \\
$\mathrm{~V}(2)$ & $4 i$ & $0.4123(3)$ & 0 & $0.5753(4)$ & $0.014(2)$ & $0.009(2)$ & $0.011(2)$ & 0 & $0.006(1)$ & 0 \\
$\mathrm{~V}(3)$ & $4 i$ & $0.3249(2)$ & 0 & $0.2168(3)$ & $0.009(1)$ & $0.019(1)$ & $0.012(1)$ & 0 & $0.0067(9)$ & 0 \\
$\operatorname{Te}(1)$ & $4 i$ & $0.26881(8)$ & 0 & $0.6940(1)$ & $0.0070(5)$ & $0.0095(5)$ & $0.0127(5)$ & 0 & $0.0057(4)$ & 0 \\
$\operatorname{Te}(2)$ & $4 i$ & $0.06743(8)$ & 0 & $0.8550(1)$ & $0.0088(5)$ & $0.0090(5)$ & $0.0087(5)$ & 0 & $0.0048(4)$ & 0
\end{tabular}

$\mathrm{Te}_{4} \mathrm{~V}_{4.64}$, monoclinic, $C 12 / \mathrm{ml}$ (No. 12), $a=13.566(3) \AA$, $b=3.930$ (1) $\AA, c=9.390(2) \AA, \beta=132.59(3)^{\circ}, V=368.6 \AA^{3}, Z=2$, $R(F)=0.026, R_{\mathrm{W}}\left(F^{2}\right)=0.062$.

Table 1. Parameters used for the X-ray data collection

$\begin{array}{ll}\text { Crystal: } & \text { silvery, irregular, size } 0.008 \times 0.038 \times 0.125 \mathrm{~mm} \\ \text { Wavelength: } & \text { Mo Ka radiation }(0.71073 \AA) \\ \mu: & 210.78 \mathrm{~cm}^{-1} \\ \text { Diffractometer: } & \text { STOE IPDS } \\ \text { Scan mode: } & \text { oscillation mode, } 200 \text { exposures, } \Delta \varphi=1^{\circ} \\ T_{\text {measurement: }} & 293 \mathrm{~K} \\ 2 \theta_{\text {max: }} & 51.6^{\circ} \\ N(h k)_{\text {unique: }} & 400 \\ \text { Criterion for } I_{0}: & I_{0}>2 \sigma\left(I_{0}\right) \\ \text { N(param })_{\text {refined: }} & 31 \\ \text { Programs: } & \text { SHELXS-86, SHELXL-93 }\end{array}$

\section{References}

1. Dronskowski, R.; Simon, A.; Plambeck-Fischer, P.: Verfeinerung der Kristallstrukturen von TasAs4 und monoklinem $\mathrm{Nb}_{4.7} \mathrm{Te}_{4}$. Z. Kristallogr. 198 (1992) 101-110.

2. Grönvold, F.; Hagberg, O., Haraldsen, H.: A Study of the Vanadium Tellurides. Acta Chem. Scand. 12 (1958) 971-982.

3. Grønvold, F.; Kjekshus, A.; Raaum, F.: The Crystal Structure of TisTe4. Acta Crystallogr. 14 (1961) 930-934.

4. Sheldrick, G. M.: SHELXS-86, Phase Annealing in SHELX-90: Direct Methods for Large Structures. Acta Crystallogr. A46 (1990) 467-473.

5. Sheldrick, G. M.: SHELXL-93. Program for refining crystal structures. University of Göttingen, Germany 1993. 